Research article Open Access

\title{
CTLA-4 +49A/G and CT60 gene polymorphisms in primary Sjögren syndrome
}

\author{
Jacques-Eric Gottenberg ${ }^{1}$, Pascale Loiseau², Mariam Azarian², Chun Chen², Nicolas Cagnard³, \\ Eric Hachulla ${ }^{4}$, Xavier Puechal ${ }^{5}$, Jean Sibilia ${ }^{6}$, Dominique Charron ${ }^{2}$, Xavier Mariette ${ }^{1}$ and \\ Corinne Miceli-Richard ${ }^{1}$
}

\begin{abstract}
${ }^{1}$ Rhumatologie, Institut Pour la Santé et la Recherche Médicale (INSERM) U802, Université Paris-Sud 11, Hôpital Bicêtre, 78 rue du Général Leclerc, Assistance Publique-Hôpitaux de Paris (AP-HP), 94275 Le Kremlin Bicêtre, France 2INSERM 396, Immunologie et Histocompatibilité, Hôpital Saint-Louis, 1 avenue Claude-Vellefaux, AP-HP, 75475 Paris cedex 10, France ${ }^{3}$ Institut Cochin, Unité de biologie moléculaire, Hôpital Cochin, 27 rue du Faubourg Saint-Jacques, 75679 Paris cedex 14, France ${ }^{4}$ Médecine Interne, Hôpital Claude Huriez, 2 avenue Oscar Lambret, 59000 Lille, France ${ }^{5}$ Rhumatologie, Hôpital du Mans, 194 avenue Rubillard, 72037 Le Mans, France ${ }^{6}$ Rhumatologie, Hôpital Hautepierre, 1 avenue Molière, 67098 Strasbourg, France
\end{abstract}

Corresponding author: Xavier Mariette, xavier.mariette@bct.aphp.fr

Received: 23 Nov 2006 Revisions requested: 9 Jan 2007 Revisions received: 5 Feb 2007 Accepted: 6 Mar 2007 Published: 6 Mar 2007

Arthritis Research \& Therapy 2007, 9:R24 (doi:10.1186/ar2136)

This article is online at: http://arthritis-research.com/content/9/2/R24

(c) 2007 Gottenberg et al.; licensee BioMed Central Ltd.

This is an open access article distributed under the terms of the Creative Commons Attribution License (http://creativecommons.org/licenses/by/2.0), which permits unrestricted use, distribution, and reproduction in any medium, provided the original work is properly cited.

\begin{abstract}
CTLA-4 encodes cytotoxic T lymphocyte-associated antigen-4, a cell-surface molecule providing a negative signal for T-cell activation. CTLA-4 gene polymorphisms have been widely studied in connection with genetic susceptibility to various autoimmune diseases, but studies have led to contradictory results in different populations. This case-control study sought to investigate whether CTLA-4 CT60 and/or +49A/G polymorphisms were involved in the genetic predisposition to primary Sjögren syndrome (pSS). We analysed CTLA-4 CT60 and $+49 \mathrm{~A} / \mathrm{G}$ polymorphisms in a first cohort of 142 patients with pSS (cohort 1) and 241 controls, all of Caucasian origin. A replication study was performed on a second cohort of 139 patients with pSS (cohort 2). In cohort 1, the CTLA-4 +49A/ $\mathrm{G}^{*} \mathrm{~A}$ allele was found on $73 \%$ of chromosomes in patients with pSS, compared with $66 \%$ in controls ( $p=0.036$; odds ratio (OR) $1.41,95 \%$ confidence interval (Cl) 1.02 to 1.95 ). No difference in CTLA-4 CT60 allelic or genotypic distribution was observed between patients $(n=142)$ and controls $(n=241)$. In
\end{abstract}

the replication cohort, the CTLA-4 $+49 \mathrm{~A} / \mathrm{G}^{\star} \mathrm{A}$ allele was found on $62 \%$ of chromosomes in patients with pSS, compared with $66 \%$ in controls ( $p=0.30$; OR $0.85,95 \% \mathrm{Cl} 0.63$ to 1.16 ). Thus, the CTLA-4 +49A/G*A allele excess among patients from cohort 1 was counterbalanced by its under-representation in cohort 2. When the results from the patients in both cohorts were pooled $(n=281)$, there was no difference in CTLA-4 $+49 \mathrm{~A} / \mathrm{G}$ allelic or genotypic distribution in comparison with controls. Our results demonstrate a lack of association between CTLA-4 CT60 or $+49 \mathrm{~A} / \mathrm{G}$ polymorphisms and $\mathrm{pSS}$. Premature conclusions might have been made if a replication study had not been performed. These results illustrate the importance of casecontrol studies performed on a large number of patients. In fact, sampling bias may account for some contradictory results previously reported for CTLA-4 association studies in autoimmune diseases.

\section{Introduction}

Polymorphisms in CTLA-4, the gene encoding cytotoxic T lymphocyte-associated antigen-4, have been widely studied in connection with genetic susceptibility to various autoimmune diseases [1], but studies have led to contradictory results in different populations.
Among CTLA-4 gene polymorphisms, a $\mathrm{G}$ to $\mathrm{A}$ transition at position $49(+49 A / G)$ of exon 1 leads to an alanine to threonine amino acid substitution at codon 17 in the leader peptide (A17T), and a $\mathrm{C}$ to $\mathrm{T}$ transition at position 60 (CT60) is located within the 3'-untranslated region [2]. The $\mathrm{G}$ allele of $+49 \mathrm{~A} / \mathrm{G}$ has been associated with a predisposition to many

$\overline{\mathrm{Cl}}=$ confidence interval; $\mathrm{CTLA}-4$ = cytotoxic T lymphocyte-associated antigen-4; OR = odds ratio; $\mathrm{pSS}=$ primary Sjögren syndrome; SNP = single nucleotide polymorphism. 
autoimmune diseases (reviewed in [1]). Both polymorphisms are in linkage disequilibrium, which warrants haplotype analysis in studies of CTLA-4 polymorphisms. The CT60 $\mathrm{G}$ allele has been reported to increase susceptibility to several autoimmune diseases [2], and a functional approach provided evidence for lower mRNA levels associated with the CT60 G allele [2].

Downie-Doyle and colleagues have recently reported a significant association of the CTLA-4 $+49 \mathrm{~A} / \mathrm{G}^{*} \mathrm{~A}$ allele and of the CTLA-4 $+49 A / G^{*} A$ allele carrier haplotypes with primary Sjögren syndrome (pSS), especially in patients with anti-SSA or anti-SSB antibodies, in a study including 111 Australian patients with pSS and 156 controls [3].

The aim of our study was to investigate in a large case-control study whether CTLA-4 CT60 and/or +49A/G SNPs were involved in genetic predisposition to pSS in French patients.

\section{Materials and methods Patients}

A first cohort of 142 unrelated patients with pSS diagnosed in accordance with the European American consensus group criteria [4] (37\% without autoantibodies, 30\% with anti-SSA antibodies only, and 33\% with both anti-SSA and anti-SSB antibodies) and 241 healthy blood donors were genotyped for CTLA-4 CT60 and +49A/G polymorphisms. A second independent cohort of 139 patients with pSS was further genotyped for CTLA-4 +49A/G polymorphisms in a replication study. The geographical origin and the clinico-biological characteristics of the patients in this second cohort were the same as those in the first. In this second cohort, $27 \%$ of patients were anti-SSA and anti-SSB negative, 35\% had anti-SSA only and $38 \%$ had both anti-SSA and anti-SSB. All patients and controls were Caucasians and provided informed consent.

\section{Genotyping}

After the isolation of genomic DNA from peripheral blood mononuclear cells, CTLA-4 CT60 and +49A/G polymorphisms were genotyped by restriction fragment length polymorphism with the use of $\mathrm{Bbvl}(+49 \mathrm{~A} / \mathrm{G})$ and Nlalll (CT60).

\section{Statistical analysis}

Allelic and genotypic frequencies of CTLA-4 CT60 and +49A/ $\mathrm{G}$ polymorphisms were compared between patients and controls by using a two-sided $\chi^{2}$ test. All genotyped SNPs were in Hardy-Weinberg equilibrium. CTLA-4 (+49A/G or CT60) haplotypes, constructed with the PHASE program, were also examined for association with pSS. $P<0.05$ was considered significant.

\section{Results}

In the first cohort of patients with pSS, the A allele of the CTLA-4 +49A/G polymorphism was found on $73 \%$ of chromosomes in patients with pSS, in comparison with $66 \%$ in controls ( $p=0.036$, odds ratio (OR) 1.41, 95\% confidence interval (Cl) 1.02 to 1.95; Table 1). No significant difference in CTLA-4 +49A/G*A allele frequencies was observed among subgroups of patients according to their anti-SSB and/or antiSSA status (Table 1). No difference in CTLA-4 CT60 allelic or genotypic distribution was observed between patients ( $n=$ $142)$ and controls $(n=241)$. CTLA-4 $(+49 A / G$ or CT60) haplotype distribution mirrored the CTLA-4 $+49 \mathrm{~A} / \mathrm{G}^{*} \mathrm{~A}$ allele excess among patients with pSS (A/A 48\%, A/G 26\%, G/G 26\%, G/A 0.4\%; in comparison with A/A 45\%, A/G 21\%, G/ G $34 \%$ among controls), leading to an excess of $+49 A / G^{\star} A$ allele carrier haplotypes among patients $(p=0.03$, OR 1.41, $95 \% \mathrm{Cl} 1.02$ to 1.95$)$.

To avoid the possibility of a false positive association of CTLA$4+49 \mathrm{~A} / \mathrm{G}^{\star} \mathrm{A}$ with pSS as a result of the somewhat small sam-

Table 1

Allellic frequencies of CTLA-4 49A/G polymorphism among patient controls

\begin{tabular}{|c|c|c|c|c|c|c|c|c|}
\hline \multirow[t]{2}{*}{$C T L A-4+49 A / G$} & \multicolumn{6}{|c|}{ Allele frequencies } & \multirow[t]{2}{*}{$p$} & \multirow[t]{2}{*}{ Odds ratio $(95 \% \mathrm{Cl}$} \\
\hline & pSS & SSA+ and SSB+ & SSA+ only & Ac+ & $\mathrm{AcO}$ & Controls $(n=241)$ & & \\
\hline Cohort 1 & $n=142$ & $n=47$ & $n=43$ & $n=90$ & $n=52$ & & & pSS vs controls \\
\hline A (Thr) & $208(73)$ & $68(72)$ & $61(71)$ & $129(72)$ & $79(76)$ & $318(66)$ & 0.036 & $1.41(1.02-1.95)$ \\
\hline G (Ala) & $76(27)$ & $26(28)$ & $25(29)$ & $51(28)$ & $25(24)$ & $164(34)$ & 0.036 & $0.70(0.51-0.98)$ \\
\hline Cohort 2 & $n=139$ & $n=52$ & $n=49$ & $n=101$ & $n=38$ & Controls $(n=241)$ & & \\
\hline A (Thr) & $173(62)$ & $59(57)$ & $66(67)$ & $125(62)$ & $48(63)$ & $318(66)$ & NS & $0.85(0.62-1.15)$ \\
\hline $\mathrm{G}$ (Ala) & 105 (38) & $45(43)$ & 32 (33) & $77(38)$ & $28(37)$ & 164 (34) & NS & $1.17(0.86-1.60)$ \\
\hline Total & $n=281$ & $n=99$ & $n=92$ & $n=191$ & $n=90$ & Controls $(n=241)$ & & \\
\hline A (Thr) & $381(68)$ & $127(64)$ & $127(69)$ & $254(66)$ & $127(71)$ & $318(66)$ & NS & $1.08(0.84-1.40)$ \\
\hline G (Ala) & 181 (32) & $71(36)$ & $57(31)$ & $128(34)$ & $53(29)$ & $164(34)$ & NS & $0.92(0.71-1.19)$ \\
\hline
\end{tabular}

Numbers in parentheses are percentages. pSS, primary Sjögren syndrome; Ac+, presence of anti SSB and/or anti-SSA; Ac0, absence of antiSSA or anti-SSB antibody; Cl, confidence interval; NS, not significant. 
ple size of our first cohort, and because the CTLA-4 +49A/ $\mathrm{G}^{\star} A$ allele has been only marginally associated with autoimmune diseases compared with the $C T L A-4+49 A / G^{*} G$ allele [1], we performed a replication study on a second independent cohort of 139 patients with pSS. In this second cohort, the CTLA-4 $+49 \mathrm{~A} / \mathrm{G}^{\star} \mathrm{A}$ allele was found on $62 \%$ of chromosomes in patients with pSS, compared with $66 \%$ in controls ( $p=$ 0.30 ; OR $0.85,95 \% \mathrm{Cl} 0.63$ to 1.16 ; Table 1 ). Thus, the CTLA-4 +49A/G*A allele excess among patients with pSS from the first cohort was counterbalanced by its under-representation in the second cohort. When the results from the patients in both cohorts were pooled $(n=281)$, there was no difference in CTLA-4 +49A/G polymorphism allelic or genotypic distribution in comparison with controls $(p=0.53$, OR $1.09,95 \% \mathrm{Cl} 0.84$ to 1.4 ; Table 1$)$. The sex ratios among patients (0.97) and controls (0.06) were different. We therefore investigated $C T L A-4+49 \mathrm{~A} / \mathrm{G}$ polymorphism genotypic distribution among males and females in the control group and found that it was not statistically different $(p=0.1)$, thus excluding any possible gender effect.

Our results therefore demonstrate a lack of association between CTLA-4 CT60 or +49A/G polymorphisms and pSS among Caucasians.

\section{Discussion}

The results from our first cohort were very close to those from the study of Downie-Doyle and colleagues [3], with a significant association of pSS with the $+49 A / G^{*} A$ allele and with the $+49 \mathrm{~A} / \mathrm{G}^{*} \mathrm{~A}$ allele carriers haplotypes. The association observed in the first cohort, of two haplotypes bearing the same allele ( $\left.C T L A-4+49 A / G^{\star} A\right)$, was actually more probably due to the statistical weight of the CTLA-4 $+49 A / G^{*} A$ allele than to a true functional effect of two different haplotypes, bearing either CTLA-4 CT60*C or CTLA-4 CT60*T alleles, each having opposite functional effects on CTLA-4 mRNA expression [2].

In fact, our results suggest a false positive association of CTLA-4 +49A $/ \mathrm{G}^{\star} \mathrm{A}$ allele with $\mathrm{pSS}$ in the first cohort of patients. When data were pooled (cohorts 1 and 2), no significant association was found with the CTLA-4 $+49 \mathrm{~A} / \mathrm{G}$ polymorphism in our Caucasian population of patients with pSS. This was not the consequence of different origin or different clinico-biological characteristics of the patients from the two cohorts and could only be the result of a sampling bias. Indeed, the findings observed in our first cohort of patients, as those from Downie-Doyle and colleagues [3], were unexpected because there are only rare examples of association of the $C T L A-4 \mathrm{~A} / \mathrm{G}^{\star} \mathrm{A}$ allele with autoimmune diseases [5-7]. Consequently, we might have made premature conclusions if a replication study had not been performed.

\section{Conclusion}

Our study illustrates the necessity to include a large number of patients in genetic case-control studies. In fact, sampling bias may partly account for some contradictory results previously reported for CTLA-4 association studies in autoimmune diseases.

\section{Competing interests}

The authors declare that they have no competing interests.

\section{Authors' contributions}

JEG contributed to the study design, performed the statistical analysis and drafted the manuscript. PL and DC supervised genotyping and contributed to DNA samples collection. MA and $\mathrm{CC}$ performed genotyping. NC performed PHASE analyses. EH, XP, and JS contributed to DNA samples collection. $\mathrm{XM}$ and $\mathrm{CMR}$ supervised the study design and gave valuable advice to JEG and PL. All authors read and approved the final manuscript.

\section{Acknowledgements}

This study was supported by Réseau de recherche clinique INSERM.

\section{References}

1. Kristiansen OP, Larsen ZM, Pociot F: CTLA-4 in autoimmune diseases - a general susceptibility gene to autoimmunity? Genes Immun 2000, 1:170-184.

2. Ueda H, Howson JM, Esposito L, Heward J, Snook H, Chamberlain G, Rainbow DB, Hunter KM, Smith AN, Di Genova G, et al.: Association of the T-cell regulatory gene CTLA4 with susceptibility to autoimmune disease. Nature 2003, 423:506-511.

3. Downie-Doyle S, Bayat N, Rischmueller M, Lester S: Influence of CTLA4 haplotypes on susceptibility and some extraglandular manifestations in primary Sjogren's syndrome. Arthritis Rheum 2006, 54:2434-2440.

4. Vitali C, Bombardieri S, Jonsson R, Moutsopoulos HM, Alexander EL, Carsons SE, Daniels TE, Fox PC, Fox RI, Kassan SS, et al.: Classification criteria for Sjogren's syndrome: a revised version of the European criteria proposed by the American-European Consensus Group. Ann Rheum Dis 2002, 61:554-558.

5. Djilali-Saiah I, Schmitz J, Harfouch-Hammoud E, Mougenot JF, Bach JF, Caillat-Zucman S: CTLA-4 gene polymorphism is associated with predisposition to coeliac disease. Gut 1998 43:187-189.

6. Suppiah V, O'Doherty C, Heggarty S, Patterson CC, Rooney M, Vandenbroeck K: The CTLA4+49A/G and CT60 polymorphisms and chronic inflammatory arthropathies in Northern Ireland. Exp Mol Pathol 2006, 80:141-146.

7. Hadj Kacem H, Bellassoued M, Bougacha-Elleuch N, Abid M, Ayadi $\mathrm{H}$ : CTLA-4 gene polymorphisms in Tunisian patients with Graves' disease. Clin Immunol 2001, 101:361-365. 